\title{
Single-shot self-interference incoherent digital holography using off-axis configuration
}

\author{
Jisoo Hong and Myung K. Kim* \\ Department of Physics, University of South Florida, Tampa, Florida 33620, USA \\ ${ }^{*}$ Corresponding author: mkkim@usf.edu
}

Received October 2, 2013; revised October 27, 2013; accepted November 5, 2013; posted November 6, 2013 (Doc. ID 198828); published November 27, 2013

\begin{abstract}
We propose a single-shot incoherent holographic imaging technique that adopts self-interference incoherent digital holography (SIDH) with slight tilt of the plane mirror in the optical configuration. The limited temporal coherence length of the illumination leads the guide-star hologram of the proposed system to have a Gaussian envelope of elliptical ring shape. The observation shows that the reconstruction by cross correlation with the guide-star hologram achieves better quality than the usual propagation methods. Experimentally, we verify that the hologram and $3 \mathrm{D}$ reconstruction can be implemented incoherently with the proposed single-shot off-axis SIDH. @ 2013 Optical Society of America

OCIS codes: (090.1995) Digital holography; (030.0030) Coherence and statistical optics; (100.3010) Image reconstruction techniques.

http://dx.doi.org/10.1364/OL.38.005196
\end{abstract}

Holographic imaging is well known for retrieving both the amplitude and phase information of an object [1]. Ironically, though the use of coherent illumination led to the realization of viable holographic imaging, it also restricted the wide usage of holographic imaging. The possibility of incoherent holography was studied in the literature [2-4], but it was not able to achieve an acceptable quality of hologram until recently. With the development of digital electronic devices and computer science over the past decade, many interesting techniques have been proposed to acquire holographic information under incoherent illumination [5-9]. Among them, the approach that uses self-interference shows feasible performance for practical applications [7-9]. Basically, it separates the light from the object into two paths and lets those beams from the same object point interfere with each other. However, the spatial incoherence of the light from the object washes out the fringe of the recorded intensity image. Instead, the complex hologram can be computationally retrieved from three or four phase-shifted images. The completely incoherent imaging process of those techniques has widened the application of holographic imaging. Rosen and Brooker have shown that a $3 \mathrm{D}$ profile of the fluorescence object can be obtained by using this approach [7]. Recently, our group reported the successful achievement of holographic recording and reconstruction of the natural outdoor scene with a holographic camera based on self-interference incoherent digital holography (SIDH) [10]. However, the use of phase shifting remains an issue that still restricts the application. The phase shifting requires the object to be nearly stationary for multiple exposures. Hence, the temporal resolution is sacrificed and it is not appropriate for high-speed imaging. Moreover, because the amount of phase shifting varies according to the wavelength of the illumination source, a large number of exposures is required for the full-color imaging. For the case of our holographic camera, we used eight phaseshifted images to create one full-color hologram [10]. In the conventional holography, the phase shifting could be removed by many techniques such as parallel phase shifting [11], Fizeau interferometry [12], the fractional
Talbot effect [13], and the random-phase reference wave [14]. However, those techniques require the illumination and the reference wave to be carefully manipulated. On the other hand, in the holographic system based on selfinterference, Kelner et al. had proposed the method to record the incoherent hologram by single shot using the spatial-light modulator (SLM) [15]. In this scheme, one of the separated beam paths is configured similar to the $4-f$ system (hence the propagation direction is inverted), while the other path does not alter the propagation. The two separated beams from a single object point interfere with opposite propagation directions, resulting in the off-axis interference.

In this Letter, we present a simple single-shot SIDH scheme using the configuration shown in Fig. 1. Basically, the optical setup of the proposed technique is almost the same as the conventional SIDH setups of $[9,10]$ except that the plane mirror M1 is slightly tilted from the on-axis position. Different from the result of [15], which records a Fourier hologram, our method

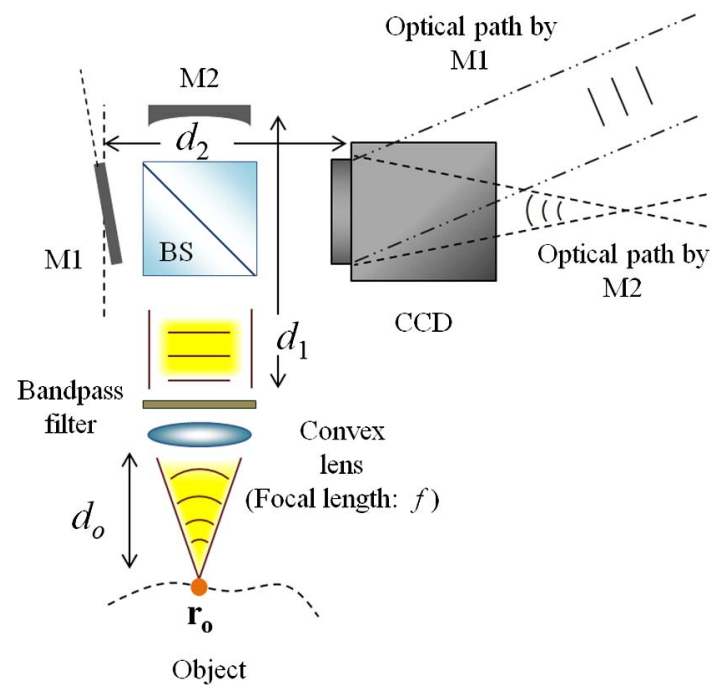

Fig. 1. Optical setup of the proposed off-axis SIDH system. BS, beam splitter; M1, plane mirror with tilt; M2, curved mirror. 
records the hologram in the form of a Fresnel hologram, meaning an off-axis hologram with finite object distance. Moreover, compared to the setup in [15], our setup requires the combination of different mirrors only once after the separation of beams, hence being easier and more flexible in implementing the system.

Similar to SIDH, the proposed scheme creates the interference only by two copies of beams emanated from the same point of the object. If the illumination is coherent, the intensity of interference corresponding to one point of the object will be

$$
u(\mathbf{r})=A\left(\mathbf{r}_{\mathbf{o}}\right)\left|Q\left(\mathbf{r}, \mathbf{r}_{\mathbf{o}}\right)+L(\mathbf{r} ; \mathbf{n})\right|^{2},
$$

where $Q$ is a quadratic phase function and $L$ is a linear phase function that is induced by the tilt of M1 ( $\mathbf{n}$ is the normal vector to the surface of M1). Here, $\mathbf{r}_{\mathbf{0}}$ and $\mathbf{r}$ denote the points on the object and CCD sensor, respectively. $A$ is a complex amplitude that is related to the intensity of the object point. However, the interference of two low coherence fields has a Gaussian envelope that depends on the amount of phase difference between two interfering beams [16]. Hence, the entire interferogram exhibiting responses from all the object points will be

$$
U(\mathbf{r})=\iint_{\mathbf{r}_{\mathbf{o}}} A\left(\mathbf{r}_{\mathbf{o}}\right)\left(2+P(\mathbf{r})\left[Q \bullet L^{*}+Q^{*} \bullet L\right]\right) \mathrm{d} \mathbf{r}_{\mathbf{o}},
$$

where $P(\mathbf{r})$ represents the envelope function incorporating the short temporal coherence length of the illumination source (or the object point itself for the selfluminous case) and the optical path difference caused by the tilt of mirror M1. $P(\mathbf{r})$ is expressed by

$$
P(\mathbf{r})=\exp \left[-\gamma \cdot \Delta(\mathbf{r})^{2}\right] .
$$

Here, $\Delta(\mathbf{r})$ is the phase difference between two copies of beams at $\mathbf{r}$, and $\gamma$ is a constant related to the temporal coherence length of the illumination. The in-phase line $\Delta(\mathbf{r})=0$ comes from the intersection of the inclined plane wavefront reflected from M1 and the spherical wavefront from M2. Hence the shape of $P(\mathbf{r})$ will look like a part of the elliptical ring.

An example of the interferogram obtained from a point source object is presented in Fig. 2(a). The optical setup was configured following the setup shown in Fig. 1 with $d_{0}=80 \mathrm{~mm}, d_{1}=200 \mathrm{~mm}$, and $d_{2}=330 \mathrm{~mm}$. The focal lengths of the convex lens and curved mirror M2 were 100 and $600 \mathrm{~mm}$, respectively. The center wavelength of LED used for illumination was $625 \mathrm{~nm}$. We inserted an additional lens (focal length: $100 \mathrm{~mm}$ ) in front of the CCD to give flexibility to the setup, and the CCD (Thorlab's DCU223M; resolution, $1024 \times 768$; pixel pitch, $4.65 \mu \mathrm{m} \times 4.65 \mu \mathrm{m}$ ) was $130 \mathrm{~mm}$ distant from the lens. We used the same configuration for all the experiments conducted throughout this Letter except for $d_{0}$. As in usual off-axis holographic imaging, a +1 or -1 order spectral component can be extracted in the angular spectrum domain with the appropriate filter. The inverse Fourier transform of the extracted spectral component gives the complex hologram of the object as shown in Figs. $\underline{2(\mathrm{c})}$ and $2(\mathrm{~d})$.

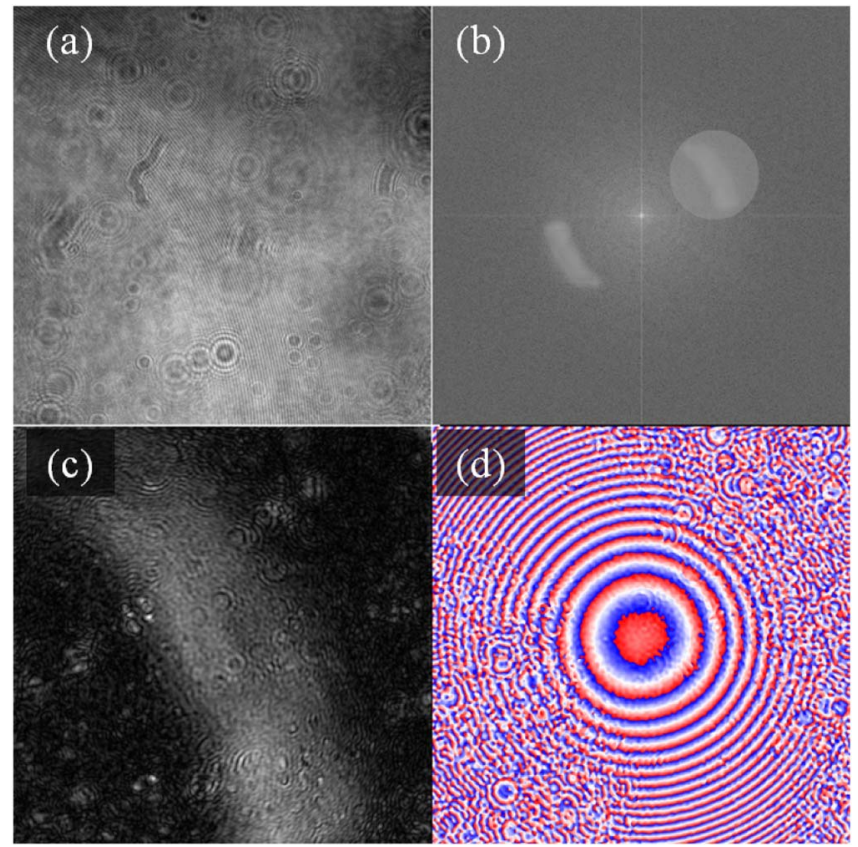

Fig. 2. (a) Interferogram $(700 \times 700)$ obtained by the point source object and (b) its angular spectrum. The brighter circle is a filter used for extracting +1 order. (c) Amplitude and (d) phase of the retrieved complex hologram.

We can apply the usual propagation methods such as angular spectrum or Fresnel propagation to this retrieved complex hologram to obtain the reconstructed image of the object. However, the short temporal coherence length restricts the valid phase information of this complex hologram inside an area where the envelope function is larger than noise. As a result, the complex hologram of the point source object is an imperfect quadratic phase function as shown in Fig. 2(d). If we consider this complex hologram as a guide-star hologram of the given system, a cross correlation with it will be another option for the reconstruction method:

$$
I(\mathbf{r})=h_{r} \odot h_{g}^{*},
$$

where $I(\mathbf{r})$ is the reconstructed image, $h_{r}$ is the retrieved complex hologram, and $h_{g}$ is the guide-star hologram. The operator $\odot$ represents the convolution. For easy variation of the reconstruction plane, the synthetic guide-star hologram can be created using Eq. (2). The $\gamma$ in Eq. (3) should be determined in a way resulting in the best reconstruction result. More details regarding a general discussion of this method can be found in [9].

We performed a preliminary experiment with the extended object to investigate the feasibility of the proposed off-axis SIDH scheme. The complex hologram of the object, which is a part of resolution target (elements 5 and 6 of group 1), was retrieved by the whole process of off-axis SIDH as explained above. The related information (recorded CCD image, angular spectrum, and the complex hologram) is provided in Fig. 3. From the retrieved complex hologram, we use various reconstruction methods (angular spectrum, Fresnel propagation, and cross correlation with guide-star hologram) to obtain the object image at the best focused plane. 


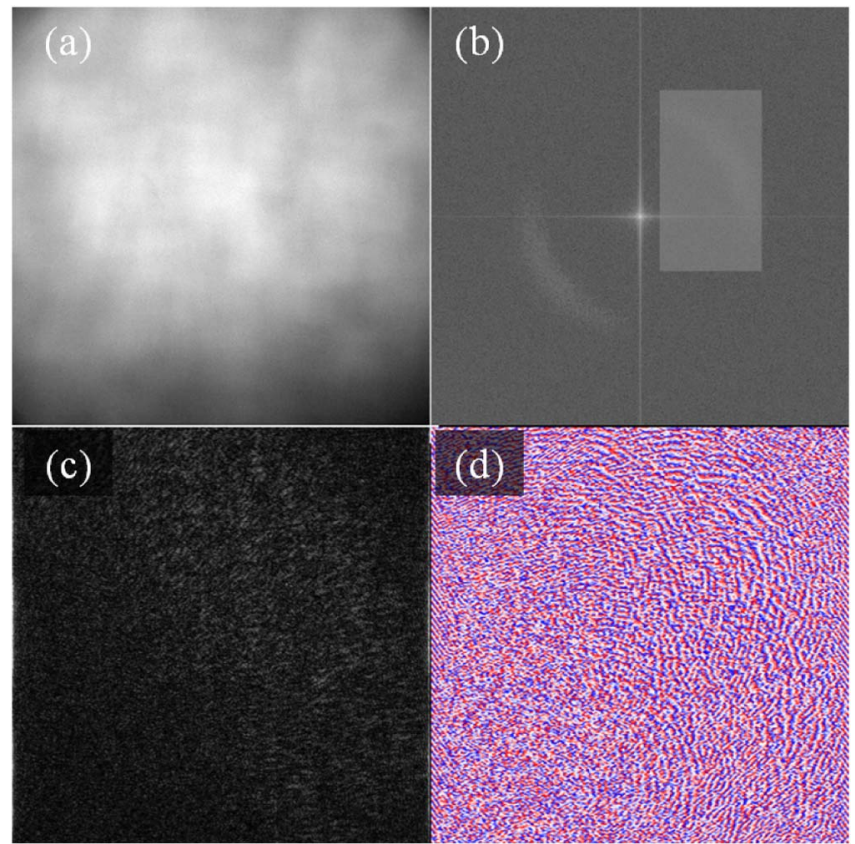

Fig. 3. (a) Interferogram $(700 \times 700)$ obtained from the extended object illuminated by LED (wavelength: $625 \mathrm{~nm}$ ) and (b) its angular spectrum. The brighter rectangle is a filter used for extracting +1 order. (c) Amplitude and (d) phase of the retrieved complex hologram.

Figure $\underline{4}$ compares the reconstruction results by three propagation methods.

Figures $5(\mathrm{a})-5(\mathrm{c})$ show the reconstructions of the point source object at the best focused plane by various reconstruction methods: (a) angular spectrum method, (b) Fresnel propagation, and (c) cross correlation with guide-star hologram. Those reconstruction results correspond to the point spread function (PSF) of each reconstruction method, which is supposed to be an impulse response for the best image quality. We can observe that there will be little difference in the PSF if we apply the cross correlation with guide-star hologram for the reconstruction. As shown in Fig. 5(d), along axis 1, the unexpected ripples at the left-hand side of the peak are suppressed by the cross-correlation method. However, for the extended object, the quality of reconstruction is significantly improved by the cross correlation with the guide-star hologram as shown in Fig. 4 . For easy comparison, we provide the average of y-cut intensity profiles of reconstruction results in Fig. 4 [see Fig. 5(g)]. This is because the complex hologram of the extended
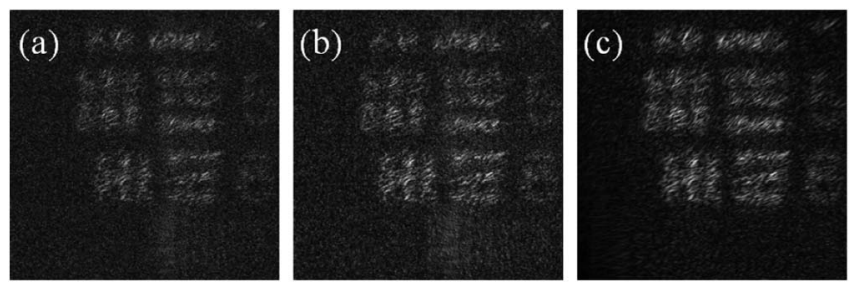

Fig. 4. Reconstruction results from the complex hologram shown in Fig. 3 by using various reconstruction methods: (a) angular spectrum, (b) Fresnel propagation, and (c) cross correlation with the guide-star hologram. The synthetic guide-star hologram was used for (c).


Fig. 5. PSF of the reconstruction computed from the complex hologram shown in Fig. 2 by using various reconstruction methods: (a) angular spectrum, (b) Fresnel propagation, and (c) cross correlation with the guide-star hologram. The images are $111 \times$ 111 area cropped from the whole FOV. (d) and (e) show the normalized intensity profile along axes 1 and 2, respectively. [Yellow, intensity profile of (a); green, that of (b); red, that of (c)]. The direction and range of axes 1 and 2 are presented as yellow lines in (a), (b), and (c). (f) compares the $y$-cut intensity profiles of Fig. 4(a) (yellow), (b) (green), and (c) (red). Each graph was obtained by averaging the $y$-cut intensities inside the range from $x=180$ to 274 . (g) illustrates the boundaries of the range as two yellow lines overlaid on Fig. 4(a).

object becomes indispensably noisier as the number of nonzero point sources at the object plane increases. This result coincides with the discussion in [9]: the presence of noise (or aberration) makes the cross correlation with the guide-star hologram more effective. And the shape of the PSFs shown in Figs. 5(a)-5(c) is asymmetric because the valid phase information in the corresponding complex hologram is restricted inside the narrow band related to the envelope function as shown in Fig. 2(d).

There are two ways to address this issue. One is to use a band-pass filter with narrower bandwidth (the bandpass filter used for experiment has $10 \mathrm{~nm}$ bandwidth). Considering Fig. 2(d), fivefold or more narrower bandwidth will recover the phase information in the entire field of view (FOV). The other way is to adopt SLM, which can implement the tilt of the plane mirror in the same plane with the curved mirror. In adopting SLM, the system parameters should be carefully designed to avoid the higher-order diffractions inside the FOV.

The ability to change the focus of the reconstruction result is one of the important features of the holography to obtain 3D information of the object. For the cross correlation with the guide-star hologram, computational 

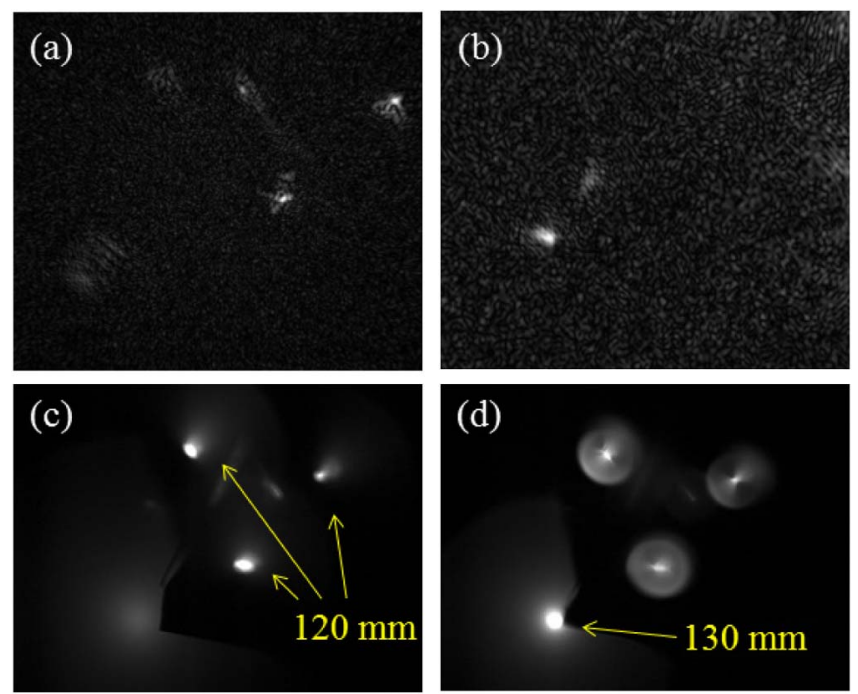

Fig. 6. Experimental results for the object composed of four LEDs located at different distances (three LEDs at $d_{0}=$ $120 \mathrm{~mm}$; one LED at $d_{0}=130 \mathrm{~mm}$ ). (a) and (b) are reconstruction results obtained from the complex hologram retrieved using the proposed off-axis SIDH. (a) Best focus at three LEDs. (b) Best focus at the fourth LED. The continuous focus change between (a) and (b) is provided as a movie file (Media 1). (c) and (d) are directly recorded images of the object. For (c), the focus is at three LEDs while (d) is at the fourth LED. In (c) and (d), the $d_{0}$ value (distance between the object and the input aperture) for each LED is provided to show the positional relation more clearly.

refocusing can be achieved by using the guide-star hologram corresponding to the point source located at the target focal plane. To show the refocusing ability, we have performed the experiment with the object composed of a set of LEDs located at different distances. As shown in Fig. 6, three LEDs are located at nearly the same distance while the fourth LED is located around $10 \mathrm{~mm}$ farther from the input aperture of the system. For the reconstruction, we used the synthetic guide-star holograms corresponding to the point sources located at different target planes. Clearly, the reconstruction results in Figs. 6(a) and 6(b) show that the proposed scheme can successfully achieve computational refocusing. We also provide a movie file that shows the continuous change of focus in computational reconstruction (Media 1).

In summary, we have shown that single-shot incoherent holography can be realized by the SIDH configuration with the slight tilt of the plane mirror. To the best of our knowledge, this is the first successful image reconstruction from the incoherent digital hologram recorded as a form of Fresnel hologram. The proposed method has an advantage that the setup is very simple and flexible. The off-axis interference between two copies of beams works similarly to the usual off-axis holography; hence, by extracting a +1 or -1 order spectral component, a complex hologram can be retrieved. However, because of the short temporal coherence length of the illumination source, the guide-star hologram of the system has a reduced area of visible interference. We have shown that the cross correlation with the guide-star hologram is the optional reconstruction method. We are expecting that the use of a band-pass filter with narrower bandwidth or SLM will much improve the reconstruction result by making the shape of the guide-star hologram closer to the perfect quadratic phase function.

The research reported in this publication was supported by the National Eye Institute of the National Institutes of Health under Award No. R21EY021876. The content is solely the responsibility of the authors and does not necessarily represent the official views of the National Institutes of Health.

\section{References}

1. D. Gabor, Nature 161, 777 (1948).

2. A. W. Lohmann, J. Opt. Soc. Am. 55, 1555 (1965).

3. G. Cochran, J. Opt. Soc. Am. A 56, 1513 (1966).

4. F. Dubois, L. Joannes, and J.-C. Legros, Appl. Opt. 38, 7085 (1999).

5. T.-C. Poon, Adv. Imaging Electron Phys. 126, 329 (2003).

6. Y. Li, D. Abookasis, and J. Rosen, Appl. Opt. 40, 2864 (2001).

7. J. Rosen and G. Brooker, Nat. Photonics 2, 190 (2008).

8. G. Pedrini, H. Li, A. Faridian, and W. Osten, Opt. Lett. 37, 713 (2012).

9. M. K. Kim, Appl. Opt. 52, A117 (2013).

10. M. K. Kim, Opt. Express 21, 9636 (2013).

11. T. Tahara, Y. Ito, Y. Lee, P. Xia, J. Inoue, Y. Awatsuji, K. Nishio, S. Ura, T. Kubota, and O. Matoba, Opt. Lett. 38, 2789 (2013).

12. D. Abdelsalam, B. Yao, P. Gao, J. Min, and R. Guo, Appl. Opt. 51, 4891 (2012).

13. M. A. Araiza-Esquivel, L. Martínez-León, B. Javidi, P. Andrés, J. Lancis, and E. Tajahuerce, Appl. Opt. 50, B96 (2011).

14. T. Nomura and M. Imbe, Opt. Lett. 35, 2281 (2010).

15. R. Kelner, J. Rosen, and G. Brooker, Opt. Express 21, 20131 (2013).

16. J. W. Goodman, Statistical Optics (Wiley, 1985). 